\title{
"MERCOESCÉPTICOS". LOS PARTIDOS POLÍTICOS OPOSITORES AL MERCOSUL EN LA ARGENTINA Y URUGUAI (1991-2006)
}

\author{
"MERCOESCÉPTICOS". POLITICAL PARTIES OPPOSED TO MERCOSUR \\ IN ARGENTINA AND URUGUAY (1991-2006)
}

Hugo Daniel Ramos ${ }^{(*)}$

Universidad Nacional del Litoral, Santa Fé (SF), Argentina.

\begin{abstract}
Resumen: El artículo analiza las posiciones de los partidos políticos en la Argentina y Uruguay, que en diferentes momentos, defendieron concepciones definidos como "mercoescépticas" (contraria al proceso de integración). En un período definido por el Tratado de Asunción y el Parlamento del MERCOSUR (1991-2006) la instalación es a la vez demuestran los cambios en las colocaciones PJ, FA, PN y PC como convertir en una posición de rechazo por parte del MERCOSUR último trabajo dos partidos. El artículo se basa en el análisis de documentos de los partidos y discusiones dentro de los Congresos Nacionales de ambos países.
\end{abstract}

Palavras-chave: MERCOSUL; partidos políticos; mercoescepticismo.

Abstrac: This work analyzes the positioning of political Parties of Argentina and Uruguay which, at different times, held conceptions defined as "mercoescépticas" (both, contrary to the integration process). We show changes in the positioning of the PJ, FA, PN and PC as well as a positioning of rejection to the Mercosur from this last two Political Parties, in a period defined by the Asunción Treaty and the installation of Mercosur Parliament (1991-2006). The work is based on the analysis of party documents and discussions within the National Congresses of both countries.

Keywords: MERCOSUL; Political parties; Mercoescepticismo.

(*) Doctor, Profesor en la Facultad de Humanidades y Ciencias, Universidad Nacional del Litoral, Santa Fe, Argentina. E-mail: <ramoshugo78@yahoo.com.ar>. Recibido en 31.10.2013, aceptado en 18.11.2013. 


\section{INTRODUCCIÓN}

El interés inicial que motivó el presente trabajo se vincula con la práctica ausencia de investigaciones que aborden a los partidos políticos en relación con el MERCOSUR. Así, y en base a una investigación doctoral ya finalizada, analizamos a un conjunto de organizaciones partidarias de Argentina y Uruguay a las que calificamos de "mercoescépticas". El uso de este concepto obedece a un explícito intento de vincular y explicar el posicionamiento de estos partidos en relación con el de sus homólogos pertenecientes a países miembros de la Unión Europea que sostienen una visión particular ("euroescepticismo") sobre el proceso de integración europeo.

Basándonos en esta idea partimos del supuesto de que pueden entenderse como "mercoescépticas" a todas aquellas organizaciones político-partidarias que: a) no apoyan la pertenencia de sus respectivos estados al proceso de integración ó, b) aceptando esa pertenencia, se oponen al avance y/o la profundización de la integración en el marco del MERCOSUR.

En relación con los partidos seleccionados para el análisis cabe mencionar que acercamientos previos a la temática nos permitieron identificar a aquellas organizaciones que a lo largo del período bajo análisis sostuvieron en determinados momentos una mirada crítica o contraria al MERCOSUR ${ }^{(1)}$. Así, consideramos al Partido Justicialista (PJ) por Argentina, y al Frente Amplio (FA), Partido Nacional (PN) y Partido Colorado (PC) por Uruguay.

El trabajo se basa en dos tipos de fuentes: documentos elaborados por los partidos políticos, en particular plataformas y programas partidarios ${ }^{(2)}$ y los debates parlamentarios al momento de aprobarse lo que denominamos "tratados fundacionales" del MER$\operatorname{COSUR}^{(3)}$.

\section{LOS PARTIDOS Y LA INTEGRACIÓN REGIONAL COMO PROBLEMA}

La noción de "partidos políticos" ha sido objeto de múltiples definiciones. Esquemáticamente, esta diversidad es resultado de dos factores relacionados entre sí: la perspectiva teórica que se adopta a la hora de analizar a los partidos políticos y el hecho de

(1) Nos referimos a nuestra tesis doctoral dedicada al análisis del posicionamiento de los partidos políticos de Argentina, Paraguay y Uruguay frente al MERCOSUR en el período 1991-2006. La periodización parte de la firma del Tratado de Asunción (1991) y se cierra con la instalación del Parlamento del MERCOSUR (2006) bajo el supuesto de que esta nueva institución inauguraba una dinámica política diferente. La tesis fue defendida en febrero del corriente año en la Facultad de Ciencia Política y Relaciones Internacionales, Universidad Nacional de Rosario.

(2) Elaborados en ocasión de las elecciones presidenciales nacionales desarrolladas en ambos países a lo largo del período bajo estudio. En el caso argentino, las elecciones de los años 1995, 1999 y 2003. En el caso uruguayo, las elecciones de 1994, 1999 y 2004.

(3) Aquellos Tratados que dieron forma y le otorgaron su fisonomía específica al proceso de integración regional. Nos referimos al Tratado de Asunción (1991), al Protocolo de Ouro Preto (1994), al Protocolo de Ushuaia (1998), al Protocolo de Olivos (2002) y al Protocolo Constitutivo del Parlamento del MERCOSUR (2005). 
que los referentes empíricos del concepto han variado sus atributos de forma considerable a lo largo de su desarrollo histórico ${ }^{(4)}$ (CINGOLANI, 2006).

En nuestro trabajo partimos de la definición de WARE (1996): "una institución que a) busca tener influencia sobre el aparato del Estado, generalmente a través de cargos en el gobierno, $y$ b) suele consistir en más de un interés de la sociedad, procurando agregar varios de ellos". La principal ventaja de esta conceptualización es que se enfoca en lo que, a nuestro criterio, constituyen los dos aspectos definitorios de los partidos: su orientación hacia el Estado y su rol de intermediación entre el Estado y la Sociedad Civil. Adicionalmente, nos permite avanzar en relación con su organización interna asumiendo que no constituyen instituciones homogéneas, ya sea en relación con su grado de fraccionalización como con su nivel de institucionalización.

Considerando estos antecedentes, retomamos el planteo de Alcántara Saez y FREIDENBERG (2003) quienes sostienen que:

el comportamiento de un partido puede observarse (...) en dos ámbitos: uno interno al partido y otro externo a él y, a su vez, este último puede manifestarse en tres arenas de actuación diferentes: el partido como organización electoral, el partido como organización de gobierno y el partido en la legislatura". El ámbito interno del partido comprende "por un lado, los individuos y en su caso, grupos que integran el partido, y por otro, las estructuras, normas y organismos creados para la toma de decisiones, el gobierno y la dirección de la organización partidista" (ALCÁNTARA SAEZ; FREIDENBERG, 2003, p. 16).

En lo referente al ámbito externo, en nuestro trabajo tuvimos en cuenta tanto al partido como organización electoral como al partido en la legislatura.

Tal como plantean FERNÁNDEZ LUZURIAGA y SOTO ROMERO (2007, p. 30) "en materia de relaciones internacionales (...) raramente se encuentran estudios sobre el rol de los partidos políticos en las políticas exteriores nacionales". Al nivel de los procesos que implica en la integración la situación es similar: "está por establecerse en qué medida los procesos de integración han afectado en lo específico y programático los mecanismos y las redes de intermediación utilizadas por los partidos en la última década" (DÁVILA, 1998, p. 160).

Basados en estas premisas, en nuestro trabajo adherimos a la propuesta de Luzuriaga (1999) quien define tres aspectos o dimensiones desde las cuales puede analizarse la relación partidos políticos/procesos de integración: en primer lugar, la dimensión movilizadora de la opinión pública (que involucra la formulación de propuestas y de programas electorales y que concibe a los partidos como organizaciones de intermediación entre el Estado y la sociedad civil); en segundo lugar, la dimensión parlamentaria (que implica concebir las tareas parlamentarias como acciones íntimamente vinculadas a la pertenencia partidaria de los legisladores, además de considerar las capacidades de los

(4) La bibliografía reciente coincide en afirmar las importantes transformaciones que éstos han experimentado en las últimas décadas, ya sea en función de sus relaciones con el Estado y la sociedad civil (Katz y Mair, 2004) o en términos organizativos (Panebianco, 2009), impactando sobre sus capacidades y las funciones que hasta el momento parecían cumplir en el seno de los respectivos sistemas políticos nacionales. 
Congresos para incidir en la política exterior de un Estado) y; en tercer lugar, la dimensión de transnacionalización partidaria directa (con un doble registro: la participación en Parlamentos Regionales de los legisladores nacionales y la membresía en organizaciones interpartidarias internacionales por parte de los partidos políticos).

Con excepción de la tercera de las dimensiones mencionadas, que en este trabajo no consideramos, la propuesta - complementaria de la categorización de ALCÁNTARA SAEZ y FREIDENBERG (2003) — nos permitió avanzar en el análisis de nuestro objeto de estudio.

\section{LOS “MERCOESCÉPTICOS": CAMBIOS Y CONTINUIDADES EN EL PERÍODO 1991-2006}

El MERCOSUR nació a partir de la firma del Tratado de Asunción (TA) entre Argentina, Brasil, Paraguay y Uruguay en el año $1991^{(5)}$. El Tratado se inscribió en el marco de la integración entre los dos primeros países ${ }^{(6)}$, al que pronto se sumaron Uruguay y Paraguay.

El TA fue firmado por cuatro presidentes que se encontraban en los primeros años de sus respectivos mandatos. Con diferencias derivadas de las particularidades nacionales, los cuatro iniciaron o consolidaron a nivel doméstico importantes procesos de reforma que abarcaron diversos ámbitos: desde la reformulación del rol del Estado en la esfera económica-social hasta el desmantelamiento casi absoluto de las barreras al intercambio comercial con el resto del mundo, en lo que dio en llamarse la "liberalización" de las economías (BOUZAS, 2002).

En términos político-partidarios, el MERCOSUR contó con un amplio consenso a nivel regional. El indicador más nítido en este sentido es el apoyo prácticamente unánime que registró al interior de los Congresos Nacionales, donde legisladores de distintas procedencias y orientaciones políticas votaron a favor del Tratado. Esto es válido para los cuatro casos nacionales, con la única excepción del Congreso uruguayo, donde tres representantes del Frente Amplio votaron en contra. Aunque extrema, la posición de estos legisladores era coherente con las discusiones al interior del Frente que motivó la firma del TA. De hecho, el partido en su conjunto expresó sólo un "apoyo crítico" al MERCOSUR. Así, durante gran parte de los años 90 el FA contó con un sector minoritario que rechazaba frontalmente al proceso de integración mientras que la amplia mayoría de sus sectores internos se debatía entre la difícil posición de criticar los "contenidos" del MERCOSUR y la defensa de la integración latinoamericana como principio.

(5) De acuerdo a DEVLIN; ESTEVADEORDAL (2001) el MERCOSUR se basó en el modelo del regionalismo abierto. Esquemáticamente éste se caracteriza por el énfasis en la liberalización comercial intra-bloque, el rol subordinado del Estado frente a los actores privados, la baja densidad institucional y su apertura al comercio internacional.

(6) Iniciado a mediados de los años 80, este proceso conoció diversos hitos, entre los que cabe destacar la firma de la "Declaración de Iguazú" (1985), el "Acta para la Integración Argentino-Brasileña" (1986), el "Acta de Amistad Argentino-Brasileña. Democracia, Pazy Desarrollo" (1986) y el "Tratado de Integración, Cooperación y Desarrollo entre la Argentina y Brasil" (1988). 
El caso del FA, sin embargo, no fue único. También hacia el interior de las principales organizaciones políticas argentinas diversos sectores se mostraron reacios a apoyar un proceso que privilegiaba la apertura comercial por sobre otros mecanismos de integración ${ }^{(7)}$. El caso del Partido Justicialista es en este sentido particularmente notorio dado que, al igual que el FA, ha sido históricamente un partido pro-integracionista ${ }^{(8)}$. Primó aquí, de todas maneras, la disciplina partidaria, y ningún legislador perteneciente al partido votó en contra de ninguno de los principales tratados del MERCOSUR.

Por su parte, el Partido Nacional y el Partido Colorado, que inicialmente apoyaron de forma entusiasta al MERCOSUR, fueron virando paulatinamente su posición. En lo año del 1998, al momento en que se discutía el Protocolo de Ushuaia ${ }^{(9)}$, el PN se manifestó en contra de introducir en el proceso integracionista "aspectos políticos" que, desde su perspectiva, no se vinculaban con los objetivos originarios del MERCOSUR. El voto negativo de todos los representantes nacionalistas en ese momento tradujo el cambio en el posicionamiento partidario, que volvería a repetirse en el futuro cuando se debatiese en torno al Parlamento del MERCOSUR (2006). De manera similar, el PC transitó decididamente el camino hacia la oposición a partir de la firma de ese mismo Protocolo que dio origen al Parlamento y con argumentos también semejantes a los empleados por el Partido Nacional.

Por su parte, el Partido Justicialista enfrentó en la primera década del siglo XXI una difícil situación: el impacto de la crisis económica, social y política experimentada en Argentina a partir del año 2001, resultado directo de las políticas neoliberales aplicadas en el país y del cual el partido había sido uno de los principales artífices. Si bien a mediano plazo la organización partidaria sortearía admirablemente la coyuntura, accediendo nuevamente al poder gubernamental en las elecciones del año 2003, lo haría al costo de su división. En relación con nuestro objeto de estudio la fractura del PJ tuvo el mérito de hacer visibles las distintas posiciones que con respecto al MERCOSUR convivían hasta entonces en el seno de la misma organización. Así, de las tres fracciones en que se fracturó el partido para las elecciones presidenciales del año 2003 sólo dos se manifestaron de forma favorable al MERCOSUR. La tercera (Frente por la Lealtad-FPL), encabezada por el ex presidente Carlos Saúl Menem, optó decididamente por el Área de Libre Comercio de las Américas (ALCA).

Las posiciones del Frente por la Lealtad-PJ, el Partido Nacional y el Partido Colorado, al igual que la del Frente Amplio y de sectores internos del Partido Justi-

(7) Así, el Diputado Antonio Cafiero, rival de la interna justicialista para las elecciones presidenciales de 1989, expresaba al momento de la aprobación del Tratado de Asunción: "esta integración que se nos plantea a través del Mercosur nos lleva a preguntarnos cuál es el tipo de integración y cuál es el resultado al que vamos a arribar con este modelo (...) ¿Esta es una integración para fortalecer un mercado latinoamericano entre los países que vamos a integrar el Mercosur? ¿Es una integración para fortalecer un camino de autonomía, de dignidad y de independencia o es una integración subordinante, que se acomoda fácilmente como una escolta al nuevo orden internacional?" (Diario de Sesiones de la Cámara de Diputados de la Nación Argentina, 14 y 15 de agosto de 1991, p. 2428-29).

(8) De acuerdo a CISNEROS; PIÑEIRO IÑíGUEZ (2002) la integración latinoamericana debe considerarse como una de las banderas históricas del peronismo junto con las de justicia social, independencia económica y soberanía política.

(9) El Protocolo de Ushuaia (1998) dio cuenta de la relevancia otorgada por los Estados participantes a la democracia, a la vez que estableció un requisito claro en relación al régimen político no sólo para los potenciales aspirantes a sumarse al proceso, sino también para la permanencia en el MERCOSUR de los Estados miembros de pleno derecho junto con los Estados asociados. 
cialista a principios de los años 90 se asemejaron en algunos aspectos. Lo que estas organizaciones discutieron en los períodos ya señalados fue, en primer lugar, los "contenidos de la integración"; a saber, si el MERCOSUR iba a ser un proceso de integración al estilo de la Unión Europea, con instituciones supranacionales, mecanismos compensatorios, y que involucrase diversas dimensiones de la vida social o si, por el contrario, iba a privilegiar sólo una integración económica-comercial. El FPL-PJ, el PN y el PC optaron por esta última opción oponiéndose a cualquier intento de profundización. Mientras el MERCOSUR se basó en el regionalismo abierto contó con su apoyo; en la medida en que la integración se redefinió en una nueva clave, sobre todo a partir del año 2003, viraron a posiciones "mercoescépticas". Por el contrario, ciertos sectores internos del Partido Justicialista y el Frente Amplio sostuvieron un "apoyo crítico" o rechazaron el proceso hasta que éste asumió mayores compromisos e incorporó nuevas dimensiones. Estos sectores y partidos son actualmente los principales defensores del MERCOSUR.

Sin embargo, los posicionamientos del FPL-PJ, PN y PC también dan cuenta del paulatino tránsito de importantes sectores partidarios de Argentina y Uruguay al campo de la oposición al MERCOSUR en sentido estricto, a favor de otras opciones integracionistas.

En los próximos apartados analizamos los posicionamientos de cada una de las organizaciones partidarias mencionadas para finalizar con una breve conclusión sobre el futuro del MERCOSUR.

\section{EL CASO DEL FRENTE AMPLIO: "MÁS INTEGRACIÓN" Y "MENOS LIBERALIZACIÓN"}

La firma del TA generó fuertes debates al interior del FA. Desde sus orígenes el Frente se había definido como latinoamericanista y mantenía una posición favorable a la integración regional ${ }^{(10)}$. Sin embargo, el MERCOSUR se construía sobre bases muy caras a las propuestas del partido. En efecto, su énfasis en los aspectos económico-comerciales y en particular su propuesta de liberalización, lo vinculaba directamente con las políticas gubernamentales neoliberales que se estaban aplicando en el plano doméstico, a las cuales el partido se oponía frontalmente.

Los distintos agrupamientos que componían el Frente manifestaron tanto posturas de apoyo crítico como de rechazo absoluto al Tratado de Asunción. La primera posición fue sostenida por los Partidos Comunista, Socialista y la Vertiente Artiguista mientras que la segunda fue defendida principalmente por el Movimiento de Participación Popular.

(10) El FA sostenía la necesidad de repensar la inserción internacional del país. Al respecto proponía la "definición y promoción de un nuevo concepto de integración económica latinoamericana. Denuncia de la anterior ALALC y actual ALADI" (Bases Programáticas de la Unidad, 1984, p. 19). En este sentido, el Frente entendía que la integración regional no debía ser sólo un mecanismo para incrementar el comercio o para defender la integridad de un modelo de desarrollo capitalista, por el contrario, debía ser la base "de la lucha colectiva por la liberación y la ruptura de la dependencia política, económica, social y cultural” (Bases Programáticas de la Unidad, 1984, p. 20). 
El apoyo crítico se basó en argumentos que destacaban coincidencias globales vinculadas a la integración regional junto con el desarrollo de críticas puntuales. Las coincidencias involucraban la aceptación de la tendencia hacia la conformación de bloques regionales en el mundo; la posibilidad de que el MERCOSUR oriente al Uruguay en el sentido de una mayor integración latinoamericana; la imposibilidad de que el país quedase al margen de los acuerdos argentino-brasileños; la aceptación de las dificultades que enfrentaba la economía uruguaya y la necesidad de que el propio Frente asumiese una participación activa con el objetivo de modificar los contenidos del proyecto integracionista. Y es precisamente en relación con los "contenidos" que se desarrollaban las críticas, ya sea vía la defensa de los intereses nacionales del Uruguay, ya sea vía la percepción de que el MERCOSUR se articulaba bajo los lineamientos del neoliberalismo ${ }^{(11)}$.

Esta última caracterización fue precisamente la que sirvió de sustento a las posiciones de rechazo absoluto:

esta integración es esencialmente perversa, provocará perjuicios muy graves a sectores sociales mayoritarios del país y pondrá en riesgo cierto de desintegración a nuestra nación (...) creemos que este proyecto de integración regional no sólo es repudiable por su carácter y contenidos sino que sus contradicciones lo hacen difícilmente viable en su actual formulación. (SCHONEBOHN, 1994, p. 36)

Frente al MERCOSUR era necesario levantar las banderas de la "verdadera" integración latinoamericana, plasmadas fundamentalmente en los programas partidarios ${ }^{(12)}$.

Puede observarse entonces una gradación de posiciones en el seno del Frente más que fracturas abruptas entre las distintas agrupaciones. Sin embargo, fue imposible conciliar las diferencias y el partido votó dividido al momento en que se discutieron tanto el TA (1991) como el Protocolo de Ouro Preto (1995).

Ahora bien, hacia finales de la década de los 90 el partido comienza a elaborar un discurso más homogéneo en torno al MERCOSUR, que se traducirá en una defensa cada vez más acentuada del proceso integracionista ¿Qué factores motivan el cambio? La respuesta comprende dos aspectos diferenciados pero que coadyuvarán a favor de un

(11) En la Resolución emitida por el Frente Amplio en ocasión de los debates en torno al Tratado de Asunción se expresaba: "Desde los albores de su historia el Uruguay reclama una experiencia de integración (...) dicho proceso debería tener una naturaleza integral, involucrando simultáneamente los ámbitos productivo, comercial y financiero (...) Hoy debemos asumir una postura definida ante el Tratado de Asunción y, en este sentido, el primer elemento a tener en cuenta es que dicho Tratado constituye apenas una propuesta de integración comercial a practicar — además - en base a un solo instrumento, que es la rebaja arancelaria (...) Sin embargo, no es en el Tratado — cuy a concepción amplia y flexible permitiría en el futuro mejorarlo y, sobre todo, ampliarlo - donde radican nuestras principales preocupaciones. Es, en cambio, la actitud de nuestro gobierno el fundamento central de nuestra visión crítica sobre este proceso. El efecto, el gobierno del doctor Lacalle no ha modificado en un ápice su concepción neoliberal" (Resolución Frente Amplio, 199, p. 1-2).

(12) En la Plataforma Electoral del partido presentada para las elecciones presidenciales de 1989 — previas a la firma del TA - la integración latinoamericana era considerada un pilar clave de sus propuestas desde una concepción que abarcaba múltiples dimensiones: "El proceso de integración regional latinoamericana no puede descansar exclusivamente en las ventajas económicas (...). Tampoco puede depender exclusivamente de una voluntad política que no tenga en cuenta los fundamentos y repercusiones económicas (...). Ambos factores deben conjugarse y la decidida voluntad política de integración atenderá tanto a las ventajas económicas como a las políticas, la defensa contra el imperialismo, las culturales, las educativas, las científicas y tecnológicas, las laborales, etc." (Plataforma Electoral del Frente Amplio, 198, p. 3). 
FA más "mercosureño": en primer lugar, la moderación programática del propio Frente y, en particular, de su fracción más "izquierdista", el MPP; en segundo lugar, el devenir del MERCOSUR, que se aproxima en algunos puntos a las propuestas del Frente a la vez que se evidencian los límites de un proceso basado sólo en la liberalización comercial.

En relación con el primer aspecto, retomamos las conclusiones de GARCÉ; YAFFÉ (2006, p. 111) que establecen de forma fehaciente la manera en que el Frente fue paulatinamente moderando su discurso y aproximándose al centro del espectro político, en un proceso que abarcó buena parte de los años 90:

En el plano programático (...) la izquierda pasó de un programa de inspiración dependentista, 'antiimperialista y antioligárquico', cuyos componentes centrales eran la reforma agraria, el rechazo de la deuda externa (considerada ilegítima), la nacionalización (o estatización) de la banca y del comercio exterior, a un programa 'progresista', del que han desaparecido los componentes centrales del programa frentista fundacional.

Este cambio se tradujo a nivel del MERCOSUR en una aceptación de que ésta era la vía más adecuada para la inserción internacional del país junto con una revalorización del mercado en el proceso de modernización de la economía uruguaya. Más aún, frente a la propuesta del ALCA, que hacia principios del nuevo siglo se presentaba como una posibilidad cierta, el MERCOSUR se erigía como una de las pocas alternativas viables capaz de salvaguardar el principio de integración regional latinoamericana.

En paralelo, el MERCOSUR había avanzado en temáticas cercanas a las demandas frentistas, en particular en lo que refiere a la dimensión política de la integración ${ }^{(13)}$, a la vez que se enfrentaba a una crisis que ponía en cuestión su base comercial y abría oportunidades para una redefinición del mismo a nivel regional. En Argentina, en particular, la idea de un "nuevo MERCOSUR" cobró forma en las propuestas programáticas de la coalición con más posibilidades de ganar las elecciones presidenciales de 1999. En este contexto, el Frente abandonó su posición crítica y se erigió en el principal defensor de la integración vía MERCOSUR

\section{EL CASO DEL PJ: DE LA "LIBERALIZACIÓN" A LA "PROFUNDIZACIÓN"}

En el caso del justicialismo argentino, y como mencionamos en el tercer apartado, las posiciones críticas con respecto al MERCOSUR no trascendieron el plano de la denuncia. Al momento de la aprobación del TA y, nuevamente, cuando se discutió el Protocolo de Ouro Preto, la disconformidad con el proceso de integración se manifestó sólo en los discursos de algunos legisladores, mas no en las votaciones ${ }^{(14)}$. Esto nos

(13) Unos de los ejemplos más claros en este sentido es el del Protocolo de Ushuaia. Posteriormente, la creación de instancias de coordinación política y de nuevas instituciones, sobre todo a partir del año 2003, reforzaron el viraje del MERCOSUR hacia una nueva concepción de la integración regional.

(14) Por caso, nuevamente el ahora Senador Antonio Cafiero volvió a manifestar su apoyo a la integración pero explicitando sus diferencias con el modelo de integración propuesto desde el Poder Ejecutivo. Así, planteaba que "todavía no hemos 
permite inferir que la mayoría de los miembros del partido concordaban con el proceso en marcha, incluyendo su formato básicamente comercial.

En este sentido, conviene mencionar que era claro para el partido gobernante la vinculación entre el MERCOSUR y las reformas neoliberales implementadas a nivel doméstico durante el período. Así, en la plataforma electoral para las elecciones presidenciales del año 1995 el partido consideraba al proceso integracionista como uno de los ejes de las transformaciones en marcha: "la apertura de la economía, las privatizaciones, la desregulación y la reforma del Estado, la integración en el MERCOSUR, replantean substancialmente el equilibrio interno entre las regiones y sectores que componen la Nación" (PLATAFORMA ELECTORAL PJ, 1995, p. 44), dando cuenta de que el mismo no era un fin en sí mismo sino "la plataforma para la inserción internacional de nuestro país" (PLATAFORMA ELECTORAL PJ, 1995, p. 72). Cuatro años más tarde, el candidato justicialista Eduardo Duhalde volvía a reafirmar, en ocasión de la campaña presidencial de 1999 su apoyo al modelo de integración mercosureño: "impulsaremos la consolidación del MERCOSUR y en consecuencia, continuaremos con la estrategia del regionalismo abierto" (PLATAFORMA ELECTORAL PJ, 1999, p. 34, las cursivas son nuestras).

En síntesis, durante los años 90 el justicialismo se alineó mayoritariamente a favor de una integración básicamente comercial y acorde con las prescripciones a favor de la liberalización que orientaban las políticas públicas de entonces. Sin embargo, la crisis desatada a nivel regional desde 1999 obligaría a replantear ciertas cuestiones acerca del modo en que funcionaba el MERCOSUR. A nivel del PJ, la crisis en Brasil y, fundamentalmente, la desarticulación del modelo económico interno basado en la convertibilidad operarían a favor de aquellos sectores más afines a una recuperación del rol del Estado y a una redefinición de la inserción internacional del país.

En las elecciones presidenciales del año 2003 se enfrentaron precisamente tres opciones que hasta entonces estaban subsumidas bajo la misma etiqueta partidaria: el Frente para la Victoria (FPV), el Frente por la Lealtad (FPL) y el Frente Movimiento Popular (FMP).

En relación con las propuestas referidas a la integración regional, para el PJ-FPL, la opción era clara: el ALCA resultaba más atractivo que el MERCOSUR. Así, si afirmaba que constituiremos a la Argentina en protagonista de la interrelación entre el MERCOSUR y el NAFTA para que no resulten de ninguna manera excluyentes en el camino hacia la conformación del ALCA proponía "iniciar inmediatamente las negociaciones con el gobierno de los Estados Unidos tendientes a un acuerdo de integración comercial" (PLATAFORMA ELECTORAL FPL, 2003, p. 10-11), lo que implicaba la disolución del MERCOSUR.

En clara contraposición a esta perspectiva el PJ-FMP planteaba la necesidad de "refundar la Argentina"; en ese proceso, el MERCOSUR ocupaba un lugar central. Así,

llegado a los niveles de mancomunidad que seguramente están en la base de nuestro pensamiento y de nuestra filosofía sobre el tema" y agregaba, refiriéndose a la estructura institucional delineada por el POP "estos organismos institucionales son todavía órganos intergubernamentales; es decir que no tienen ninguna dosis de transnacionalidad, de supranacionalidad. $Y$ esto (...) resulta ya insuficiente por la envergadura y la importancia que el MERCOSUR está tomando". Diario de Sesiones, Cámara de Senadores de la Nación Argentina, 5 de julio de 1995, p. 2.474). 
apelaba a "convocar de inmediato a una Cumbre de Presidentes del MERCOSUR a los fines de refundar el Mercado Regional que tiene para la Argentina un sentido estratégico" (PLATAFORMA ELECTORAL FMP, 2003, p. 15) proponiendo:

la libre circulación de personas dentro del mercado (...) que en el MERCOSUR los títulos de nivel secundario, terciario y universitario serán validos en todo el territorio del mercado (...) establecer en el MERCOSUR un salario mínimo, vital y móvil, con vigencia para los trabajadores de todo el mercado (...) la creación de la Corte Suprema del MERCOSUR (PLATAFORMA ELECTORAL FMP, 2003, p. 15-16).

Para finalizar con "la nueva constitución del MERCOSUR que (...) contemplará un gobierno republicano, democrático, social, representativo y federal” (PLATAFORMA ELECTORAL FMP, 2003, p. 16).

Por último, pero también dentro del marco del PJ, el FPV proponía “un MERCOSUR igualitario, integrado, y que nos dé el mismo marco de desarrollo que al resto de los países que lo integran" (PLATAFORMA ELECTORAL FPV, 2003, p. 5) y enunciaba su preferencia por un modelo de integración comunitario, al estilo de la Unión Europea. El eje de esta propuesta se vinculaba con la reconstrucción del Estado y con la aplicación de políticas neokeynesianas para reactivar la economía.

Es interesante en este marco rescatar las expresiones vertidas por el candidato de esta fórmula, Néstor Kirchner, (DI TELLA; KIRCHNER, 2003, p. 215-216) en una entrevista que se le realizó durante la campaña electoral:

La próxima elección presidencial es la elección de qué modelo de país queremos los argentinos: tendremos que elegir entre los que creen que la solución es el dólar y quienes sostenemos que la salida es una moneda nacional fuerte; entre los que proponen el ALCA y los que proponemos el MERCOSUR (...) mi elección es MERCOSUR.

La definición de la elección presidencial a favor de esta última fracción definiría en los próximos años la orientación del MERCOSUR, más aún si consideramos que al año siguiente el Frente Amplio asumiría la presidencia del Uruguay, sumándose así a las señales a favor de un "nuevo MERCOSUR" que también bajaban desde Brasil.

\section{EL PARTIDO NACIONAL Y EL PARTIDO COLORADO: DE LA DEFENSA A LA CRÍTICA DEL MERCOSUR}

A diferencia de los casos anteriores, donde lo que observamos es un giro favorable al proceso integracionista (Frente Amplio) o una redefinición de las relaciones de fuerzas internas favorable a los sectores que apostaron por una profundización del MERCOSUR (Partido Justicialista), en el Partido Nacional y el Partido Colorado lo que registramos es un cambio en los posicionamientos partidarios en un sentido contrario al avance del MERCOSUR.

En relación con el PN, conviene recordar que el Presidente Luis Alberto Lacalle, quien negoció el ingreso de su país al proceso integracionista, pertenecía a esta fuerza 
política. En este sentido, dos fueron los argumentos principales esgrimidos por el partido para justificar la membresía uruguaya al MERCOSUR en 1991: por un lado, el incremento de la competitividad, en el marco del proceso de reformas "modernizadoras" puestas en marcha por la administración Lacalle; por otro, la inevitabilidad del proceso, para evitar la marginación del país del contexto subregional y atemperar el impacto económico de un acuerdo preferencial que excluyese al Uruguay.

En el caso del PC, dos de los sectores que formaban parte del gobierno de Lacalle ${ }^{(15)}$, el Foro Batllista y la Lista 15, manifestaron su apoyo, mientras que otros dos sectores, uno de los cuales también participaba del gabinete, expresan algunas reservas; éstos fueron la Unión Colorada y Batllista y Cruzada 94. Un argumento común de estas últimas agrupaciones fue que el ingreso uruguayo no debía significar el abandono de otras opciones comerciales a nivel mundial mientras que Cruzada 94 añadió que era necesario además una activa participación del Estado en el proceso de adaptación y reconversión de los sectores productivos al mercado ampliado. Los sectores que sostuvieron un apoyo decisivo, en cambio, destacaron la inevitabilidad de la participación del Uruguay, compartiendo los argumentos con el Partido Nacional. Aún así, mientras la Lista 15 adhirió sin reservas a la tesis de la modernización, agregando la necesidad de una rápida apertura, el Foro Batllista sostuvo la precaución de que el proceso fuese gradual, marcando distancia con las propuestas neoliberales a ultranza ${ }^{(16)}$.

A lo largo de la década de los 90 los sectores mayoritarios del PN acentuaron su posición favorable a la implementación de políticas neoliberales en el plano doméstico ${ }^{(17)}$. En este marco, el MERCOSUR fue percibido de manera similar a como lo entendía el PJ argentino por estas fechas:

La constitución del MERCOSUR como espacio comunitario que potencie la capacidad productiva conjunta de los países de la región es una parte fundamental de esta inserción (...) en la medida en que contribuye y acompaña la búsqueda de una integración al mundo que asegure un dinamismo sostenido de la producción nacional (MANOS A LA OBRA, PN, 1994, p. 33, las cursivas son nuestras).

(15) Cabe recordar en este punto que el gobierno de Lacalle no había obtenido mayorías legislativas. Frente a esta situación, el líder nacionalista logró conformar un acuerdo interpartidario con el Partido Colorado, que fue denominado de "Coincidencia Nacional" y que le garantizó un mínimo de gobernabilidad, aunque de forma inestable. La "Coincidencia Nacional" se acordó entre los distintos sectores del partido gobernante y los sectores mayoritarios del Partido Colorado, posibilitando la incorporación de cuatro ministros colorados (pertenecientes al Foro Batllista, Unión Colorada y Batllista y Lista 15) sobre un total de doce cargos ministeriales. Los ocho ministerios restantes se dividieron entre las agrupaciones del Partido Nacional. A partir del año 1992 el Foro Batllista y la Lista 15 abandonaron el acuerdo.

(16) En este sentido, Julio María Sanguinetti expresaba "Una violenta inserción en el mundo producirá internamente un sacudón violentísimo a nuestra producción agraria e industrial, con una importante secuela de desocupación (...) el arancel externo que separe al MERCOSUR del mundo será relativamente bajo y en consecuencia no nos estaremos alejando de los grandes mercados, sino apenas manteniendo la protección imprescindible" (en SANGUINETTI; ABREU; COURIEL, 1991, p. 10-11).

(17) Es importante considerar que hasta finales de los años 80 el sector mayoritario dentro del partido era el Movimiento por la Patria, liderado por el histórico líder Wilson Ferreira Aldunate, de clara orientación de centro-izquierda (COSTA BONINO, 1996). Luego de la muerte éste en 1989 , la conducción partidaria quedó a cargo del sector liderado por Luis Alberto Lacalle, ubicado en el centro-derecha del espectro político. 
El MERCOSUR fue entendido así como un aspecto complementario de una estrategia más amplia que definía el modelo de inserción internacional del país.

Este posicionamiento, por otra parte, no excluyó la apelación a una integración 'más profunda' siempre y cuando ésta fuese funcional a ese objetivo mayor. Así para las elecciones presidenciales de 1994 "Manos a la Obra" consideraba que el MERCOSUR era "el ámbito a partir de cual sería posible 'modernizar' al país según ciertos lineamientos vinculados con la inserción competitiva en el mercado mundial", el desarrollo industrial y científico, la eficiencia de la acción estatal y "la movilización total de nuestra gente y recursos" (MANOS A LA OBRA, PN, 1994, p. 10).

La modernización vía MERCOSUR involucraba necesariamente una fuerte presencia de la dimensión económica-comercial de la integración, que ocupa el principal punto de interés de la agrupación nacionalista, pero también otros aspectos no estrictamente económicos tales como las migraciones y la necesidad de elaborar políticas educativas y culturales a nivel regional, entre otros. La agrupación resignificaba así la tradición nacionalista referida a la subregión y la redefinía en función del MERCOSUR: "Ahora es el Uruguay en la Comunidad del Mercosur y el Mundo” (MANOS A LA OBRA, PN, 1994, p. 23).

Para la misma fecha, el Foro Batllista, sector mayoritario del Partido Colorado, compartía muchos de los presupuestos del $\mathrm{PN}^{(18)}$, aunque advertía que "la necesaria apertura [económica] no debe ser ingenua e ignorar las medidas de protección de los otros Estados, especialmente los más desarrollados” (PROGRAMA 2000 DEL FORO BATLLISTA, 1994, p. 15). Su estrategia de desarrollo partía de dos orientaciones básicas: (1) El proceso de apertura externa y (2) la integración regional (PROGRAMA 2000 DEL FORO BATLLISTA, 1994, p. 122).

En este marco, si bien el Foro tampoco dejaba de lado áreas tales como la cultura y la educación, limitaba la profundización del MERCOSUR a los aspectos económico-comerciales, dejando en claro también que constituía un medio para una mejor inserción en la economía mundial:

La opción estratégica por una economía abierta impone un crecimiento que otorgue carácter esencial a las relaciones con el exterior (...) En la aplicación del Tratado del MERCOSUR y en la determinación del arancel externo común se debe reconocer que cuanto más integrado esté el país en el mundo, menor será el efecto provocado por las inestabilidades en las economías de los socios más fuertes. Esto se logra con aranceles bajos (PROGRAMA 2000 DEL FORO BATLLISTA, 1994, p. 104-105, las cursivas son nuestras).

Como puede observarse, en ambos casos está claro que el MERCOSUR es considerado como parte fundamental de las políticas económicas domésticas de carácter

(18) La vinculación que se realiza entre MERCOSUR y políticas neoliberales queda clara cuando consideramos la siguiente afirmación: "El mantenimiento y profundización del proceso de integración regional requiere que las medidas internas y las negociaciones con nuestros vecinos tiendan a: posibilitar, por un lado el crecimiento sin incertidumbres de las actividades competitivas, protegiéndolas en el marco regional de la competencia desleal de las exportaciones subsidiadas de países extrarregionales, $y$, por otro, facilitar los procesos de ajuste, incremento de eficiencia, reconversión de los sectores más afectados" (Programa 2000 del Foro Batllista, 1994, p. 123). 
neoliberal, a las que prestan su apoyo ambos partidos, aunque con diferencias ancladas en las diversas evaluaciones que ambas organizaciones realizan sobre los riesgos de la apertura económica. A su vez, ambos lo consideran como un instrumento al servicio de una estrategia de internacionalización de la economía uruguaya, un paso "intermedio" necesario para modernizar la estructura productiva del país.

Ahora bien, para finales de los años 90 el posicionamiento del Partido Nacional comienza a cambiar de forma notoria. Como mencionamos en el tercer apartado, uno de los disparadores de ese cambio fue la incorporación al acervo normativo del MERCOSUR del Protocolo de Ushuaia que, de acuerdo a los miembros de esta organización, cambiaba las "reglas de juego" y los principios sobre los cuales se había acordado construir el proceso de integración en 1991. En este sentido, las expresiones fueron muy claras al momento de discutirse el Protocolo en el Congreso uruguayo ${ }^{(19)}$ :

Consideramos que con relación a estos tratados [del MERCOSUR], que tienen un nacimiento de tipo económico de carácter regional y que implican la integración de los países que los signan por razones de tipo comercial, resulta esencial evitar que se incorporen conceptos de carácter político, como ocurre en esta ocasión (SENADOR WALTER SANTORO, PN, las cursivas son nuestras).

Este posicionamiento vuelve a reafirmarse para las elecciones presidenciales de 1999 donde el MERCOSUR es concebido única y exclusivamente en el marco del regionalismo abierto. Así, en el apartado denominado "Política de Integración" se propone como objetivo:

Mejorar la inserción externa del país bajo los principios del regionalismo abierto, que favorezcan la multiplicación de los intercambios económicos y socioculturales con exclusión de todo carácter político no derivado de compromisos asumidos convencionalmente (PROGRAMA ÚNICO DE GOBIERNO del PN, 1999, p. 08, las cursivas son nuestras).

Cabe destacar que la reafirmación del regionalismo abierto no implica todavía un claro tránsito del nacionalismo a un posicionamiento "mercoescéptico". Como mencionamos en el tercer apartado, ese paso recién se dará en la primera década del Siglo XXI al compás de la reformulación del MERCOSUR.

Por su parte, el Partido Colorado comienza también a señalar más claramente sus diferencias con el rumbo del MERCOSUR. Para las elecciones presidenciales de 1999 la propuesta del partido se sitúa en un horizonte proyectivo basado en una planificación del rol de Uruguay en el MERCOSUR sustentado en el regionalismo abierto ${ }^{(20)}$, la

(19) En la Cámara de Representantes el Protocolo de Ushuaia fue debatido y votado el 15 de diciembre de 1998. El resultado de la votación en general fue de cinco votos negativos (todos del PN) contra 58 a favor. Cabe destacar que la mayoría de los legisladores nacionalistas se habían retirado al momento de la votación. En la Cámara de Senadores, nuevamente, contó con cinco votos contrarios del PN. En esta última Cámara el Protocolo fue votado el 4 de mayo de 1999.

(20) "Reafirmamos entonces la necesidad de fortalecer una economía abierta al exterior, competitiva, integrada regionalmente, con un concepto de regionalismo abierto, para aumentar su apertura y diversificación frente al resto del mundo" (Plataforma y principios del Partido Colorado, 1999, p. 19). 
continuidad de políticas de apertura tanto a nivel regional como nacional y el perfeccionamiento de la estructura institucional regional. Si bien se recupera la instancia de crisis por la que está atravesando el MERCOSUR, ésta es percibida como coyuntural y factible de abordar mediante ajustes basados en la profundización de la integración económica-comercial ${ }^{(21)}$. Aún así, es claro que la propuesta traduce cierta disconformidad con algunos aspectos del funcionamiento del bloque, ya sea la denominada "diplomacia presidencial", en desmedro de la consolidación institucional; o la dificultad de alcanzar el estadio de zona de libre comercio por la imposición de trabas al comercio recíproco. En este sentido se plantea que es prioritario "dar estabilidad a los acuerdos alcanzados [y al] cumplimiento por todas las partes de los compromisos asumidos [para] evitar los choques provocados por la inestabilidad económica de cualquiera de los socios" (PLATAFORMA Y PRINCIPIOS DEL PARTIDO COLORADO, 1999, p. 26).

De todas formas, y al igual que para el caso del Partido Nacional, la presencia de estas críticas no indica todavía una oposición al MERCOSUR, aspecto que sí se puede registrar en el siguiente momento de nuestro análisis: las discusiones por el Protocolo Constitutivo del Parlamento del MERCOSUR.

En este sentido, el Protocolo fue debatido por un importante número de legisladores que se articularon en dos grupos de diferenciación político-partidaria: frenteamplistas vs. nacionalistas y colorados. La idea de "confrontación" resume adecuadamente el marco en el que se desarrollaron las discusiones en torno al Protocolo, en particular en la Cámara de Representantes. Claramente, el PCPM puso de manifiesto dos posicionamientos en torno a la integración latinoamericana en los partidos políticos uruguayos, que podrían resumirse en la fórmula "integración profunda" (Frente Amplio) vs "integración comercial" (Partido Nacional y Partido Colorado).

Tanto el PN como el PC se opusieron de forma contundente y categórica al Parlamento del MERCOSUR. Sus argumentaciones específicas abarcaron tres ámbitos: "razones de forma, razones de conveniencia y razones de oportunidad" (REPRESENTANTE JAIME TROBO, PN)(22). Las primeras daban cuenta de que, para estos partidos, el PCPM eran inconstitucional ${ }^{(23)}$; las segundas de que, desde su perspectiva, algunas disposiciones del Protocolo perjudicaban los intereses nacionales del país ${ }^{(24)}$; las últimas de que, dado el conflicto que por ese entonces afectaba profun-

(21) "El primer tramo del próximo gobierno (... enfrentará el desafí de responder adecuadamente a condiciones adversas en la región y en el mundo. Éstas son producto de dificultades económicas de los socios mayores del MERCOSUR; de la persistencia — aún del agravamiento — de restricciones al comercio de muchos de nuestros productos y en especial de la situación coyuntural de baja en los precios internacionales de muchos de ellos" (Plataforma y principios del Partido Colorado, 1999, p. 29).

(22) Diario de Sesiones de la Cámara de Representantes de la República Oriental del Uruguay, 21 de noviembre de 2006, p. 89.

(23) “[Es necesario] un serio análisis en cuanto a si nuestra Constitución admite y permite, sin violentarla, la creación de este Protocolo Constitutivo del Parlamento del MERCOSURy, fundamentalmente, si eso se puede encuadrar en el orden constitucional vigente en nuestro país" (Senador Gustavo Penadés, PN) Diario de Sesiones de la Cámara de Senadores de la República Oriental del Uruguay, 27 de septiembre de 2006, p. 426

(24) "Creemos que es inconveniente que el Uruguay ingrese a un proceso que no tendrá retorno, en el cual cede trozos de su soberanía y de su capacidad para autodeterminarse" (Representante Jaime Trobo, PN. Diario de Sesiones de la Cámara de Representantes de la República Oriental del Uruguay, 21 de noviembre de 2006, p. 90. 
damente las relaciones argentino-uruguayas ${ }^{(25)}$, no era el momento para avanzar con una institución de las características del Parlamento.

Ahora bien, ifueron razones de "forma, conveniencia y oportunidad" las que explican de forma suficiente el voto contrario al Parlamento por parte del Partido Nacional y del Partido Colorado? ${ }^{(26)}$ Para los legisladores del Frente Amplio la respuesta era negativa: su opinión era que, a lo que se oponían estos partidos, era a abandonar el "MERCOSUR comercial" y a empezar a transitar un "nuevo" camino en la integración regional $^{(27)}$.

Si bien los propios legisladores del PN y del PC así lo indicaron en varias oportunidades $^{(28)}$ la lectura del debate completo da cuenta de una cuestión más compleja: la percepción de que "el MERCOSUR no funciona"; de que en el MERCOSUR "no se cumple lo acordado" y de que, en definitiva, el proceso de integración "no sirve al Uruguay". Sin reflejarse en todas las intervenciones de aquellos que votaron en contra del PCPM, esta idea está presente en el suficiente número de ellas como para que sea notorio:

¿vamos a dar nuestro voto para avanzar en el proceso de integración institucional de un MERCOSUR que vive una crisis de esa naturaleza sobre la cual Brasil ha permanecido totalmente ajeno, donde los mecanismos de solución de controversias no han funcionado salvo virtualmente y donde no hemos logrado ninguna solución a nuestros problemas? Me parece que por este camino no vamos a ningún lado (REPRESENTANTE CARLOS MOREIRA, PN, las cursivas son nuestras);

En clave analítica estas expresiones, que traducen los posicionamientos expresados por el PN y el PC, darían cuenta de que el MERCOSUR perdió, hacia el año 2006, una base considerable de sustentación política en el Uruguay ${ }^{(29)}$. En efecto, si bien es

(25) En el momento en que el Protocolo llegó a la Cámara de Representantes del Uruguay el conflicto por "las papeleras" había alcanzado su punto más álgido, con el bloqueo de los puentes que comunicaban Argentina con Uruguay por parte de organizaciones ambientalistas argentinas. Este conflicto operó como un fuerte condicionamiento del debate.

(26) En la Cámara de Senadores el resultado de la votación fue de 17 votos a favor y 9 en contra (en general y en particular) mientras que en la Cámara de Representantes fue de 50 a favor y 13 en contra (votación en general) y de 52 a favor y 13 en contra (votación en particular).

(27) Al respecto, la Representante del FA Silvana Charlone planteaba: "yo tengo el convencimiento (...) de que, más allá de cualquier coyuntura, hay sectores en cuya ideología y concepción está la de que nunca, bajo ninguna circunstancia, votarían un Parlamento del MERCOSUR porque tienen una concepción de MERCOSUR comercial, que no trasciende más allá" (Diario de Sesiones de la Cámara de Representantes de la República Oriental del Uruguay, 21 de noviembre de 2006, p. 116)

(28) "Quiero que se tenga bien presente que desde el artículo único que envía el Poder Ejecutivo relativo a la constitución del MERCOSUR hasta el propio Tratado de Asunción y los subsiguientes, como el de Ouro Preto, el MERCOSUR nunca se salió de la temática comercial" (Representante Luis Lacalle Pou, PN. Diario de Sesiones de la Cámara de Representantes de la República Oriental del Uruguay, 21 de noviembre de 2006, p. 124.

(29) Las plataformas electorales presentadas por ambos partidos en las elecciones presidenciales del año 2004 anticiparon este posicionamiento. En efecto, el PC planteaba "El Mercosur constituyó más del 70\% del destino de nuestras exportaciones de bienes y servicios. Hoy participa en una cifra cercana a la mitad (...) El Mercosur se transforma así en el punto de partida para uno estrategia comercial y no en el punto de llegada" (Bases Programáticas del Partido Colorado, 2004, p. 34). En otras palabras, si bien el Partido confirmaba su proyecto de hacer del Uruguay un nodo central para las exportaciones regionales, también consideraba que el MERCOSUR "no debe significar claudicación alguna en la inserción y relacionamiento del país con el mundo globalizado” (Bases Programáticas del Partido Colorado, 2004, p. 83). Por su 
claro que algunos sectores partidarios discutieron en torno a modelos de integración (el "MERCOSUR comercial" vs. "integración profunda" defendida por el Frente Amplio), también lo es que un número no determinado de legisladores no sólo votó contra el Parlamento del MERCOSUR, sino contra el mismo MERCOSUR.

\section{CONCLUSIÓN}

A lo largo de la exposición precedente detallamos los posicionamientos de los partidos políticos argentinos y uruguayos que en determinados períodos mantuvieron una actitud crítica con respecto al MERCOSUR, discutiendo los “contenidos” de la integración.

Observamos en primer lugar los casos del Frente Amplio y de sectores del Partido Justicialista que hacia principios de los años 90 sostenían que era necesario avanzar "más allá" de los aspectos económico-comerciales. Aún con ciertas similitudes, en el plano institucional nacional ese posicionamiento se tradujo de forma diferente. Así, mientras que la mayoría del Frente Amplio sostuvo un "apoyo crítico", votando a favor de los Tratados fundacionales del MERCOSUR pero expresando sus diferencias con el modelo de integración elegido (y permitiendo que uno de sus sectores internos votara negativamente el Tratado de Asunción y el Protocolo de Ouro Preto), el Partido Justicialista mantuvo su disciplina partidaria y acalló sus diferencias internas, sosteniendo de forma unánime en el ámbito del Congreso Argentino los distintos Tratados del MERCOSUR.

La configuración de un posible "nuevo MERCOSUR", del cual el Parlamento regional constituía en ese entonces una clara expresión, contribuye a explicar el giro en el posicionamiento del Partido Nacional y del Partido Colorado, hasta entonces claros defensores del proyecto integracionista. La identificación de los aspectos que sostenían esa posición, dan cuenta de los motivos por los cuales estos partidos adoptan una actitud "mercoescéptica" a partir de entonces. Ahora bien, del análisis de las fuentes consideradas en nuestra investigación también surge que este posicionamiento se inicia en torno a la disputa de cómo debe ser la integración pero también incluye un componente no despreciable de rechazo al MERCOSUR en sentido estricto, es decir, de que estas organizaciones partidarias ya no consideran al proceso de integración como una opción viable para la inserción internacional del Uruguay.

En una mirada de largo plazo, la investigación deja en claro que durante los años 90 el MERCOSUR contó con un consenso activo (Partido Nacional, Partido Colorado, Partido Justicialista) o crítico (Frente Amplio y sectores del justicialismo argentino) que se perdió a partir del nuevo siglo, con el decisivo giro a posiciones mercoescépticas por parte del nacionalismo y del Partido Colorado uruguayo, junto al sector hasta entonces mayoritario del justicialismo argentino. En este sentido, y si bien el MERCOSUR experimentó diversas coyunturas críticas a lo largo de su primera década, la clave parece encontrarse en dos procesos que se desarrollaron simultáneamente desde finales de los años 90. Por

parte, el planteo del PN es similar: "La inserción de nuestro país en el MERCOSUR debe significar una reafirmación de nuestra vocación histórica y preferente de integración con la región pero no en forma exclusiva o excluyente con relación al continente o al mundo" (Programa para un gobierno de Compromiso Nacional, Partido Nacional, 2004, p. 77). 
un lado, y en primer lugar, la crisis que se desató en la región en el período 1999-2002, que afectó tanto la credibilidad de las políticas económicas hasta entonces implementadas como las bases sobre las cuales se asentaba el proceso de integración. Esta crisis facilitó a su vez el recambio dirigencial a nivel regional y los desplazamientos de poder entre distintos sectores partidarios (en particular en el justicialismo argentino) que permitieron relanzar el MERCOSUR a partir del año 2003 en una nueva clave.

Por otro lado, y en segundo lugar, cabe recordar que ya desde el Protocolo de Ushuaia el MERCOSUR puso en juego variables explícitamente políticas, profundizadas a partir del año 2003 en base a la ya mencionada integración "social y productiva". Es frente a esta dirección, que perfila claramente a partir de este año, cuando se hacen explícitas las posiciones contrarias al MERCOSUR en sentido estricto, de todas aquellas fuerzas políticas más comprometidas con el libre mercado, las políticas neoliberales y la liberalización a escala continental.

En fin, la investigación sugiere finalmente que los cambios en los posicionamientos partidarios, lejos de sostenerse incólumes a lo largo del período, han ido variando en función de las disputas de poder al interior de cada organización y de los avatares del propio proceso de integración. En la actual coyuntura tanto en Argentina como en Uruguay gobiernan partidos y sectores partidarios favorables a la continuidad del proceso; no está garantizado que ésta sea la situación a futuro, lo que plantea serios interrogantes acerca del sustento político-partidario del MERCOSUR en los próximos años.

\section{BIBLIOGRAFÍA}

AlCÁnTARA SAEZ, Manuel; FREIDENBERG, Flavia. Partidos Políticos de América Latina. Cono Sur. México: Fondo de Cultura Económica, 2003.

BOUZAS, Roberto (coord.). Realidades Nacionales Comparadas. Tomos I y II. Buenos Aires: Universidad Nacional de San Martín-Fundación/OSDE, 2002.

CINGOLANI, Luciana. Partidos políticos y sistemas de partidos. In: AZNAR, Luis; DE LUCA, Miguel (org.). Política. Cuestiones y problemas. Buenos Aires: Emecé, 2006.

CISNEROS, Andrés; PIÑERO IÑIGUEZ, Carlos. Del ABC al MERCOSUR. La integración latinoamericana en la doctrina y praxis del peronismo. Buenos Aires: ISEN-GEL, 2002.

COSTA BONINO, Luis. Wilson Ferreira Aldunate y la lógica nacionalista. Política Uruguaya. 1996. Disponible en: <http://www.politicauruguaya.com/uy/WFAyLLN.pdf> Consulta: 10 ago de 2009.

DÁVILA, Andrés. Partidos e Integración en América Latina. ¿Tienen la culpa de algo? Perfiles Latinoamericanos, México, v. 7, n. 12, p. 141-168, jun. 1998.

DEVLIN, Robert; ESTEVADEORDAL, Antoni. ¿Qué hay de nuevo en el nuevo regionalismo de las Américas? INTAL-ITD-STA, Working Paper, n. 7, Buenos Aires, ago. 2001.

DI TELLA, Torcuato; KIRCHNER, Néstor. Después del derrumbe. Teoría y práctica política en la Argentina que viene. Buenos Aires: Galerna, 2003.

FRENTE AMPLIO. Bases programáticas de la unidad. Montevideo, 9 de agosto de 1984.

Plataforma electoral del frente amplio. Montevideo, 4 de junio de 1989.

Resolución plenario nacional frente amplio. Montevideo, 27 de abril de 1991.

GARCE, Adolfo; YAFFE, Jaime. La izquierda uruguaya: ideología, estrategia y programa (19712004). Revista América Latina Hoy, n. 44, p. 87-114, 2006. 
HARTLEB, Florian. A Thorn in the Side of European Elites: the new euroscepticism. Brussels: Centre for European Studies, 2011.

KATZ, Richard; MAIR, Peter. El partido cartel: la transformación de los modelos de partidos y de la democracia de partidos. Zona Abierta, n. 108-109, p. 9-42, 2004.

PANEBIANCO, Angelo. Modelos de partido. Madrid: Alianza Editorial, 2009.

PARTIDO COLORADO. Programa 2000 del foro batllista. El uruguay entre todos. Montevideo, 1994. . Plataforma y principios del partido colorado. Por el uruguay y su gente. Montevideo, septiembre de 1999.

. Bases programáticas del partido colorado. Montevideo, 24 de agosto de 2004.

PARTIDO JUSTICIALISTA. Plataforma electoral PJ. Capital Federal, 6 de abril de 1995. . Plataforma electoral PJ. Ciudad de Buenos Aires, 11 de agosto de 1999.

. Plataforma electoral frente por la lealtad. Ciudad de Buenos Aires, 3 de marzo de 2003. . Plataforma electoral frente movimiento popular. Ciudad de Buenos Aires, 8 de marzo de 2003. Plataforma electoral frente por la victoria. Ciudad de Buenos Aires, 22 de abril de 2003.

PARTIDO NACIONAL, Montevideo, 12 de agosto de 2004.

. Manos a la obra. Cómo hacerlo. Montevideo, octubre de 1994.

Programa único de gobierno del partido nacional. Montevideo, 10 de septiembre de 1999.

. Resolución directorio partido nacional. Montevideo, 18 de septiembre de 2006.

PEREZ ANTÓN, Romeo. Los partidos políticos y el MERCOSUR: el caso uruguayo. Cuadernos del CLAEH, v. 22, n. 77, p. 7-37, 1997.

RAMOS, Hugo. La dimensión partidaria de la integración regional. Los partidos políticos de Argentina, Paraguay y Uruguay frente al MERCOSUR (1991-2006). 2013. Tesis (Doctorado en Relaciones Internacionales) — Universidad Nacional de Rosario, Rosario (inédita).

. Algunos comentarios en torno a las modificaciones actuales en el sistema de partidos argentino (1983-2011). Revista Estudios, Córdoba, n. 26, p. 81-94, jul./dez. 2011.

SALUDJIAN, Alexis. Hacia otra integración sudamericana. Críticas al MERCOSUR neoliberal. Buenos Aires: Libros del Zorzal, 2004.

SANGUINETTI, Julio; ABREU, Sergio; COURIEL, Alberto. Uruguay y el MERCOSUR, Cuadernos de la Integración, Montevideo, v. 1, n. 1, 1991.

SCHONEBOHM, Dieter. MERCOSUR: ¿desafío o amenaza? Gobierno, partidos, empresarios y sindicatos ante la integración. Montevideo: FESUR, 1994.

WARE, Alan. Partidos políticos y sistemas de partidos. Madrid: Istmo, 1996. 